\title{
Microwave ablation of autonomously functioning thyroid nodules: a comparative study with radioactive iodine therapy on the functional treatment success
}

\author{
Mehmet Sercan Erturk ${ }^{11,2}$, Bulent Cekic ${ }^{3}$, Mehmet Celik4, Isil Demiray Uguz \\ ${ }^{1}$ Division of Endocrinology and Metabolism, Department of Internal Medicine, University of Health Sciences, \\ Antalya Training and Research Hospital, Antalya, Turkey \\ ${ }^{2}$ Division of Endocrinology and Metabolism, Department of Internal Medicine, Izmir Katip Celebi University, \\ Ataturk Training and Research Hospital, Izmir, Turkey \\ ${ }^{3}$ Department of Radiology, University of Health Sciences, Antalya Training and Research Hospital, Antalya, Turkey \\ ${ }^{4}$ Division of Endocrinology and Metabolism, Department of Internal Medicine, Trakya University Medical Faculty, Edirne, Turkey \\ ${ }^{5}$ Department of Nuclear Medicine, University of Health Sciences, Antalya Training and Research Hospital, Antalya, Turkey
}

\begin{abstract}
Introduction: The objective of this study was to compare the efficiency of microwave ablation (MWA) and radioactive iodine (RAI) in the treatment of toxic adenoma (TA), and to investigate the functional treatment success of the used modalities for its remission.

Material and methods: Treatment outcomes — thyroid hormone levels and nodule characteristics — of 30 patients (23:7 F:M; $52.77 \pm$ 11.13 years) treated by MWA were compared with the those of 35 patients ( $24: 11 \mathrm{~F}: \mathrm{M} ; 61.43 \pm 12.60$ years) treated by RAI. The baseline characteristics of TAs, which are gender and pre volume, were analogous and did not show any statistical significance $(p>0.05)$. Thyroid hormone levels of patients treated with two different methods were measured after 9 months, and the obtained results were compared. Results: Although there was no statistically significant difference in the nodule volume $(p>0.05)$, there was a greater volume reduction rate $(\mathrm{VRR} \%)$ in the group treated with MWA rather than RAI $(\mathrm{p}<0.05)$ at the end of the follow-up. In the MWA group, there was a higher increase in FT3 than in the RAI group $(p<0.05)$. Furthermore, no statistically significant difference in TSH $(p=0.124)$ and FT4 $(p=0.144)$ levels of the patients as treatment outcomes was observed. The therapeutic success was accomplished in 18/30 $(60 \%)$ of the MWA group and in 24/35 (68.6\%) of the RAI group.

Conclusions: Therapeutic success of MWA and RAI did not show any statistically significant difference $(p=0.471)$. However, the development of hypothyroidism in 7 of 35 patients treated with RAI was observed. On the other hand, no case of post treatment hypothyroidism was observed in patients treated with MWA. In this regard, MWA could be a great alternative to RAI due to its advantages in terms of non-exposure to radiation and lower risk of post-treatment hypothyroidism. (Endokrynol Pol 2021; 72 (2): 120-125)
\end{abstract}

Key words: thyroid; nodule; toxic adenoma; microwave ablation; thyroid hormones

\section{Introduction}

The second most widespread cause of hyperthyroidism after Graves' disease is autonomously functioning thyroid nodules (AFTN) [1]. Toxic adenoma (TA) and toxic multinodular goitre take place as an outcome of focal and/or diffuse hyperplasia of thyroid follicular cells [2]. AFTN can lead to the development of a series of functional abnormalities, including euthyroidism and overt hyperthyroidism [3]. Although euthyroidism can be achieved with thionamides, the symptoms of hyperthyroidism can be controlled rather than undertaking permanent treatment [4]. Antithyroid drugs (ATDs) can be an option in the long-term treatment of patients who do not want surgery or radioactive iodine (RAI) therapy [5]. The choice between radioiodine and surgery is based on individual patient factors and the regional availability of specialist thyroid surgeons. Permanent treatments for toxic thyroid adenomas are RAI treatment and surgery [6,7]. Thionamides are often used before surgery and RAI to prepare patients for permanent treatments.

Several techniques have been applied up to now for the treatment of AFTNs, some of which are percutaneous ethanol injection (PEI) [8,9] and laser ablation (LA) [10]. Due to their disadvantages such as less effectivity in the treatment of solid nodules and the requirement of multiple and tedious treatment secession for PEI and LA, respectively, alternative approaches have been tried in the last decades. In recent years, in particular, American thyroid associations have proposed radio frequency $(\mathrm{RF})$ ablation as a new thermal ablation treat- 
ment modality for toxic adenomas patients who are not suitable or refuse to undergo permanent treatment options and ATDs [11]. The risk of major complications such as recurrent laryngeal palsy and hypoparathyroidism with these techniques is very low [12]. Also, its important advantages have been considered as good alternatives for patients unwilling to undergo surgery or RAI therapy due to benefits such as acceptable nodule shrinkage, the ability to be treated on an outpatient basis, and long-term effects [13].

In recent years, alternatively, microwave ablation (MWA) has been emerging as a new technology and is attracting increasing attention for the treatment of thyroid nodules because of its advantages including good cosmetic results, low complication rate, and nodule shrinking efficacy, especially for young patients who are preferring non-surgical treatments [14]. Although MWA therapy has been applied in several studies for the treatment of benign thyroid nodules, to the best of our knowledge, no study has been conducted up to now for the examination of the effectiveness of MWA in the treatment of AFTNs. Thus, the purpose of this study is to investigate the efficacy and safety of MWA ablation for AFTN therapy in a large population of patients.

\section{Material and methods}

\section{Ethics statement}

University of Health Sciences Antalya Training and Research Hospital Ethics Committee approved this study (Ethics Committee

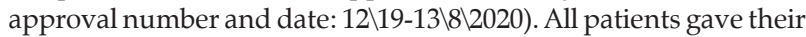
written informed consent before the procedure.

\section{Patients}

This study was carried out as a retrospective analysis of a prospectively collected data. From January 2017 to August 2020, 35 patients treated with radioactive iodine (RAI) for biochemically non-occult AFTN and 30 patients who underwent ultrasound-guided microwave ablation for biochemically non-occult AFTN were included in the study. The inclusion criteria of the MWA group were as follows: 1) patients with functioning solid thyroid nodules; 2) ineligibility or refusal to undergo surgery; and 3) benign nodules (fine needle aspiration biopsy results based on Bethesda categories). The exclusion criteria were as follows: nodules with increased risk for malignancy and malignant nodules (Bethesda categories $\geq 3$ ).

\section{Laboratory assessment}

A Unicel TM Dxl 800 Access Immunoassay System (Beckman Coulter Inc., Brea, CA, USA) was used to measure serum thyroid function tests. The normal ranges of these tests were $0.61-1.1 \mathrm{ng} / \mathrm{dL}, 2.5-3.9$ $\mathrm{pg} / \mathrm{mL}, 0.34-5.86 \mu \mathrm{IU} / \mathrm{mL}, 0-4 \mathrm{IU} / \mathrm{mL}$, and $0-10 \mathrm{IU} / \mathrm{ml}$ for free $\mathrm{T} 4$, free $\mathrm{T} 3$, thyrotropin (TSH), anti $\mathrm{Tg}$, and $\mathrm{TPO}$, respectively. Laboratory tests were performed 1,3, and 9 months after treatment modalities.

\section{Microwave ablation}

A microwave ablation system (ECO-100AI3) with a microwave generator producing $30-40 \mathrm{~W}$ of power at $2450 \mathrm{MHz}$ either continuously or in a pulse, a flexible cable, and internally-cooled 16-gauge thyroid antenna with $10 \mathrm{~cm}$ shaft length with a $3.0 \mathrm{~mm}$ active tip was used. Autonomous thyroid nodule ablation was applied on an outpatient basis under local anaesthesia without sedation. The patient was placed in a supine position with the neck mildly hyperextended. After determining the appropriate puncture side, a mixture of 30/70\% lidocaine (Osel Pharmaceuticals, Istanbul, Turkey) and saline was applied along the puncture path from the skin to the thyroid capsule and then infused into the surrounding thyroid capsule to preserve vital structures adjacent to the thyroid nodule. An internally cooled thyroid microwave ablation antenna was positioned under ultrasound guidance via trans-isthmic approach or lateral cervical approach. A moving shot technique was used to ablate the target nodule throughout the procedure. Therapy was completed when the entire nodule was covered with hyperechoic echo, which is indicative of ablation. Vital signs were monitored during the procedure, and phonation was evaluated intermittently. After the procedure, all patients were followed up for 2 hours with cold compression to the neck to prevent haematoma. Before discharging the patients, an ultrasound examination was performed to evaluate the changes in MWA-induced focal complications.

\section{RAI ablation}

Scintigraphic images were obtained in all patients 15-20 min after the application of $75 \mathrm{MBq}$ Tc-99m-pertechnetate and recorded with a scintillation camera (Mediso Nucline XR® TH / 22, Budapest, Hungary). Unifocal overactive areas with reduced or suppressed uptake in the remaining thyroid tissue were thought to be compatible with non-occult AFTN along with suppressed TSH. The patients were administered a fixed dose of $15 \mathrm{mCi}$ (555 MBq) RAI ${ }^{131}$ I without prior antithyroid therapy). Necessary information about radiation safety was given verbally and in writing, and then the patients were discharged.

\section{Treatment efficiency and follow-up}

The volumes of the nodules before, after, and during the followup process were assessed by ultrasonography examination via the use of a 5-14 MHz linear probe of a real-time ultrasound system (Aplio 500, Toshiba Medical Systems, Tokyo, Japan). The orthogonal diameters of the nodules before ablation, which are $a, b$, and c calculated from the equation $\mathrm{V}=$ wabc/6, where $\mathrm{V}=$ volume, $\varpi=3.14159, \mathrm{a}=$ the largest diameter, and $\mathrm{b}$ and $\mathrm{c}=$ the other two perpendicular diameters. In the calculation of the volume reduction ratio (VRR\%), the following equation was used:

\section{[Baseline volume - volume at 9 months] / [Baseline volume] 100}

The success of the therapy was accepted as euthyroid state at 6 months without antithyroid medical treatment. Antithyroid therapy was not used before MWA or RAI and during follow-up. Beta-blocker therapy was used if needed. With the aim of restoring euthyroidism, the levothyroxine treatment was administrated in the presence of a permanent hypothyroid state. Hypothyroidism, which is defined as high serum TSH concentration together with a low serum free $\mathrm{T} 4$ concentration, is a functional major side effect compared between two groups.

\section{Statistical analysis}

SPSS ${ }^{\circledast} 20.0$ (Statistical Packages for Social Sciences; SPSS Inc, Chicago, Illinois, USA) was used to assess all the statistical tests. The normality and the homogeneity of the data were evaluated by Shapiro-Wilk and Levene's test of homogeneity. Qualitative variables were presented as percentages or frequencies, while continues variables were reported as mean $\pm \mathrm{SD}$. The data of the two groups were compared by the use of appropriate independent t, Mann-Whitney U, Wilcoxon rank, and Pearson chi-square tests. The significance level was considered as a $p$ value of less than 0.05 . 


\section{Results}

\section{Patients' data}

The baseline characteristic data of the patients and the nodules according to their treatment grouping are summarised in Table 1. A statistical significance test was conducted to reveal the analogies between the two groups. Results revealed that gender, TSH, volume, and thyroid autoantibodies did not exhibit any statistically significant difference ( $\mathrm{p}>0.05)$. Also, before MWA therapy, positive thyroid autoantibodies were present in 4:30 patients, and 2:35 before RI therapy.

\section{Treatment outcomes}

Table 2 presents the treatment outcomes of the 6-month follow-up of the two groups. Investigation of Table 2 reveals that, at the end of the follow-up, there was no statistical difference between MWA and RI groups in terms of nodule volume ( $p=0.916)$. On the other hand, it can be clearly seen that there was a higher volume reduction rate in the MWA group compared to the RI group $(p=0.008)$. In FT3 values, there was a greater increase in the MWA group rather than RI ( $p<0.001)$. In addition, no statistically significant difference between the TSH $(p=0.124)$ and FT4 $(p=0.144)$ levels of the patients in the two groups was observed. The functional undesirable effect was $20 \%$ in the RI group in terms of clinical hypothyroidism percentage, whereas no undesirable effect was observed for the MWA group. Overall, none of the patients in both of the groups exhibited major complications in either of the groups.

The functional therapeutic success (FTS), defined as the restoration of euthyroidism, of the two groups was also investigated, and the detailed cross tabulation results are given in Table 3 , which shows that FTS was achieved in $18 / 30$ and $24 / 35$ patients with a percentage of $60.0-88.6 \%$ in the MWA and RI groups, respectively, and there was no statistically significant difference between the values of them $(p=0.471)$.

\section{Discussion}

Our study showed that MWA is effective in treating TAs. While VRR\% was higher in patients who underwent MWA, the therapeutic success rate was higher in the group receiving RAI. On the other hand, although

Table 1. The baseline features of the patients and nodules according to treatment modalities

\begin{tabular}{|c|c|c|c|}
\hline Baseline & $\operatorname{RAI}(\mathrm{n}=35)$ & MWA $(n=30)$ & p value \\
\hline Age & $61.43 \pm 12.60$ & $52.77 \pm 11.13$ & 0.005 \\
\hline Gender (F:M) & $24: 11$ & $23: 7$ & 0.467 \\
\hline FT3 $(2.5-3.9 \mathrm{pg} / \mathrm{mL})$ & $3.34 \pm 0.85$ & $4.36 \pm 0.76$ & 0.000 \\
\hline FT4 (0.61-1.1 ng/dL) & $0.96(0.78-1.10)$ & $1.12(0.89-1.51)$ & 0.006 \\
\hline TSH (0.34-5.86 $\mu \mathrm{lU} / \mathrm{mL})$ & $0.05(0.03 \pm 0.13)$ & $0.07(0.04-0.22)$ & 0.291 \\
\hline Volume (cc) & $6.08(4.24-8.82)$ & $8.08(4.03-12.50)$ & 0.130 \\
\hline Thyroid autoantibodies & & & 0.403 \\
\hline Positive:negative (\%) & $5: 30(14.3 \%)$ & $4: 26(13.3 \%)$ & \\
\hline
\end{tabular}

FT3 — free triiodothyronine; FT4 — free thyroxine; TSH — thyroid-stimulating hormone; RAI — radioactive iodine; MWA — microwave ablation; $\mathrm{p}<0.05$ is significant

Table 2. Treatment outcomes after nine months

\begin{tabular}{|c|c|c|c|}
\hline & RAI & MWA & p value \\
\hline Volume [cc] & $3.48(2.00-5.43)$ & $3.30(2.16-6.10)$ & .916 \\
\hline VRR\% & $45.81(20.60-45.81)$ & $54.29(45.23-59.61)$ & .008 \\
\hline FT3 $(2.5-3.9 \mathrm{pg} / \mathrm{mL})$ & $3.14(2.75-3.32)$ & $3.79(3.37-4.70)$ & .000 \\
\hline FT4 (0.61-1.1 ng/dL) & $0.87(0.78-1.00)$ & $0.98(0.78-1.06)$ & .144 \\
\hline TSH (0.34-5.86 $\mu \mathrm{lU} / \mathrm{mL})$ & $1.20(0.68-1.95)$ & $1.04(0.19-1.55)$ & .124 \\
\hline \multicolumn{4}{|c|}{ Functional undesirable effect } \\
\hline Hypothyroidism (\%)* & $7 / 35(20 \%)$ & 0 & \\
\hline
\end{tabular}

RAI — radioactive iodine; MWA — microwave ablation; *hypothyroidism was defined as high TSH concentration in association with low serum free thyroxine concentration; VRR — volume reduction ratio; FT3 — free triiodothyronine; FT4 — free thyroxine; TSH — thyroid-stimulating hormone; $\mathrm{p}<0.05$ is significant 


\begin{tabular}{lccc} 
& \multicolumn{2}{c}{$\begin{array}{c}\text { Functional therapeutic outcome (FTO) } \\
\text { Therapeutic unsuccess }\end{array}$} & Total \\
\hline MWA Treatment & & & \\
\hline Count & 18 & 12 & 30 \\
\hline$\%$ within MWA & $60.0 \%$ & $40.0 \%$ & $100.0 \%$ \\
\hline$\%$ within FT0 & $42.9 \%$ & 52.2 & $46.2 \%$ \\
\hline RAI Treatment & & & 35 \\
\hline Count & 24 & $31.4 \%$ & $100.0 \%$ \\
\hline$\%$ within RAI & $68.6 \%$ & $47.8 \%$ & $53.8 \%$ \\
\hline$\%$ within FTO & $57.1 \%$ & & 65 \\
\hline Total & & 23 & $100.0 \%$ \\
\hline Count & 42 & $35.4 \%$ & $100.0 \%$ \\
\hline$\%$ within Treatment & $64.6 \%$ & $100.0 \%$ & \\
\hline$\%$ within FTO & $100.0 \%$ & & \\
\hline
\end{tabular}

$\mathrm{RAI}$ - radioactive iodine; MWA - microwave ablation; chi-square test. Pearson $\chi^{2}=0.519, \mathrm{p}=0.471$

hypothyroidism did not develop in any case after MWA, the development of hypothyroidism was statistically higher in the group that received RAI in the follow-up.

Hemithyroidectomy and RAI ablation are the permanent treatment used for TAs. The surgical process lowers the risk of permanent hypocalcaemia in the presence of damage to the affected side in the parathyroid glands. Furthermore, it ensures the maintenance of thyroid function by means of contralateral healthy thyroid lobe. All in all, the risk of permanent unilateral laryngeal nerve injury after surgical procedure is still between $0 \%$ and $2.1 \%$ [15].

The other alternative form of permanent therapy is RI, due to its high percentage of clinical efficacy between 3 and 12 months and its low cost [16]. However, RAI is absolutely contraindicated in pregnant or breastfeeding women [17]. The main disadvantage of RAI is the delay in conception for at least 6 months and the development of undesirable complications after exposure to radiation in both male and female patients [18, 19].

TAs lead to the investigation of alternative treatments, especially in selected patient groups, due to the disadvantages of both surgery and RAI treatment in their permanent treatments. A new development for the treatment of thyroid diseases - ultrasound-guided MWA - has the advantages of being minimally invasive, safe, and effective [20-22]. Huo S et al. reported that there was no adverse effect on the patient or foetus in the follow-up after MWA for an autonomously functioning thyroid nodule in a pregnant patient [23]. No study comparing MWA and RA has been reported in the literature until now. However, there are studies comparing RAI with other minimally invasive methods such as radiofrequency ablation and alcohol ablation [24, 25]. As in these studies, the end points in our study to evaluate the effectiveness of each treatment were volume nodule reduction and resolution of the hyperthyroid state 9 months after RAI and MWA. When evaluating through VRR\%, the rate of volume reduction after RAI in TAs has been reported as $35-54 \%$ in the literature $[16,26,27]$. After radiofrequency ablation, this rate was reported as $52.1 \%$ and $79.7 \%$ [28, 29]. After percutaneous alcohol ablation in toxic nodules, the rate of thyroid nodule volume reduction was reported to be $66 \%$ in the $12^{\text {th }}$ month [30]. In our study, although VRR\% was evaluated at the $9^{\text {th }}$ month after MWA, it was found that it was similar to VRR\% in the $12^{\text {th }}$ month of other minimally invasive methods. However, it was determined that VRR\% was statistically higher in the MWA group compared to the patients who received RAI.

Hypothyroidism is less common after initial toxic adenoma radioiodine therapy. The risk of developing hypothyroidism after RAI is $12-32 \%$ after one year [31]. Focal nodule autonomy areas take up more radioiodine, while in the contralateral thyroid tissue suppressed by the hyperthyroid state, uptake is limited. As a result, radioiodine tends to destroy only autonomic areas, and most patients remain euthyroid after radioiodine administration [32]. Patients who develop hypothyroidism usually have incomplete suppression of iodine uptake in the extranodular tissue, or the patient has chronic lymphocytic thyroiditis with an autonomic toxic nodule $[33,34]$. In our study, when the thyroid function of patients was evaluated after treatment, euthyroidism was achieved in $68.6 \%$ of the RAI group and $60 \%$ of the MWA group.

Although the rate of euthyroid patients was high in the RAI group, hypothyroidism was observed in 
7 patients in the RAI group after treatment, while no hypothyroidism was found in the MWA group. In addition, 5 patients in the RAI group had antibody positivity (TPOAb), and all of these patients developed hypothyroidism after treatment. However, although 2 patients in the MWA group had antibody positivity, no hypothyroidism was observed after treatment. We think that this is because MWA affects only the autonomic nodule, and even if the antibody is positive, it does not destroy other healthy thyroid tissue. In a study by Cervelli R. et al. [24], although most of the patients (72\%) treated with RAI converted to euthyroidism, clinical hypothyroidism was found in 5 patients. In addition, it was determined that 5 hypothyroid patients after treatment had positive TPOAb before RAI treatment. It has been reported that high TPOAb levels may be considered as a risk factor for hypothyroidism $[35,36]$. Moreover, $\mathrm{I}^{131}$ retained by the autonomic nodule after RI treatment is taken up by the thyroid tissue surrounding the autonomic nodule and damages healthy thyroid cells [37]. Because thermal damage is created in the local area of the nodule with MWA, it does not damage healthy cells around the nodule.

The limitation of our study is that, due to the small sample size, large patient series with long-term follow-up are needed to better evaluate the efficacy after MWA treatment.

\section{Conclusion}

Although RI and MWA have similar effects in maintaining normal thyroid hormone function in patients with toxic autonomic thyroid nodules, post-treatment of RI, hypothyroidism is more common especially in patients with TPOAb(+). When it is desired to avoid hypothyroidism after treatment, MWA can be considered as a reliable method in toxic adenoma in patients with high antibody levels.

\section{Disclosure statement}

No potential conflict of interest was reported by the authors.

\section{Authors' contributions}

The study conception and design were by M.S.E, B.C, and M.C. M.S.E, B.C, M.C, and I.D.U performed the material preparation, data collection, and analysis. M.S.E wrote the first draft of the manuscript, and all authors commented on previous versions of the manuscript.

\section{Data availability statement}

The data that support the findings of this study are available on request from the corresponding author. The data are not publicly available due to privacy or ethical restrictions.

\section{References}

1. Laurberg P, Pedersen KM, Vestergaard H, et al. High incidence of multinodular toxic goitre in the elderly population in a low iodine intake area vs. high incidence of Graves' disease in the young in a high iodine intake area: comparative surveys of thyrotoxicosis epidemiology in East-Jutland Denmark and Iceland. J Intern Med. 1991; 229(5): 415-420, doi: 10.1111/j.1365-2796.1991.tb00368.x, indexed in Pubmed: 2040867.

2. Parma J, Duprez L, Van Sande J, et al. Diversity and prevalence of somatic mutations in the thyrotropin receptor and Gs alpha genes as a cause of toxic thyroid adenomas. J Clin Endocrinol Metab. 1997; 82(8): 2695-2701, doi: 10.1210/jcem.82.8.4144, indexed in Pubmed: 9253356.

3. Treglia G, Trimboli P, Verburg FA, et al. Prevalence of normal TSH value among patients with autonomously functioning thyroid nodule. Eur J Clin Invest. 2015; 45(7): 739-744, doi: 10.1111/eci.12456, indexed in Pubmed: 25940693

4. Singer PA, Cooper DS, Daniels GH, et al. Treatment guidelines for patients with hyperthyroidism and hypothyroidism. Standards of Care Committee, American Thyroid Association. JAMA. 1995; 273(10): 808-812, indexed in Pubmed: 7532241.

5. Elbers L, Mourits M, Wiersinga W. Outcome of very long-term treatment with antithyroid drugs in Graves' hyperthyroidism associated with Graves' orbitopathy. Thyroid. 2011; 21(3): 279-283, doi: 10.1089/thy.2010.0181, indexed in Pubmed: 21190446.

6. Vidal-Trecan GM, Stahl JE, Eckman MH. Radioiodine or surgery for toxic thyroid adenoma: dissecting an important decision. A cost-effectiveness analysis. Thyroid. 2004; 14(11): 933-945, doi: 10.1089/thy.2004.14.933, indexed in Pubmed: 15671772.

7. Kang AS, Grant CS, Thompson GB, et al. Current treatment of nodular goiter with hyperthyroidism (Plummer's disease): surgery versus radioiodine. Surgery. 2002; 132(6): 916-23; discussion 923, doi: 10.1067/msy.2002.128691, indexed in Pubmed: 12490836.

8. Lippi F, Ferrari C, Manetti L, et al. Treatment of solitary autonomous thyroid nodules by percutaneous ethanol injection: results of an Italian multicenter study. The Multicenter Study Group. J Clin Endocrinol Metab. 1996; 81(9): 3261-3264, doi: 10.1210/jcem.81.9.8784080, indexed in Pubmed: 8784080.

9. Mazzeo S, Toni MG, De Gaudio C, et al. Percutaneous injection of ethanol to treat autonomous thyroid nodules. AJR Am J Roentgenol. 1993; 161(4): 871-876, doi: 10.2214/ajr.161.4.8372778, indexed in Pubmed: 8372778.

10. Pacella CM, Bizzarri G, Spiezia S, et al. Thyroid tissue: US-guided percutaneous laser thermal ablation. Radiology. 2004; 232(1): 272-280, doi: 10.1148/radiol.2321021368, indexed in Pubmed: 15155898.

11. Ross DS, Burch HB, Cooper DS, et al. 2016 American Thyroid Association Guidelines for Diagnosis and Management of Hyperthyroidism and Other Causes of Thyrotoxicosis. Thyroid. 2016; 26(10): 1343-1421, doi: 10.1089/thy.2016.0229, indexed in Pubmed: 27521067.

12. Chung SR, Suh CH, Baek JH, et al. Safety of radiofrequency ablation of benign thyroid nodules and recurrent thyroid cancers: a systematic review and meta-analysis. Int J Hyperthermia. 2017; 33(8): 920-930, do i: 10.1080/02656736.2017.1337936, indexed in Pubmed: 28565997.

13. Nixon IJ, Angelos P, Shaha AR, et al. Image-guided chemical and thermal ablations for thyroid disease: Review of efficacy and complications. Head Neck. 2018; 40(9): 2103-2115, doi: 10.1002/hed.25181, indexed in Pubmed: 29684251.

14. Yue W, Wang S, Wang B, et al. Ultrasound guided percutaneous microwave ablation of benign thyroid nodules: safety and imaging follow-up in 222 patients. Eur J Radiol. 2013; 82(1): e11-e16, doi: 10.1016/j. ejrad.2012.07.020, indexed in Pubmed: 22940229.

15. Landerholm K, Wasner AM, Järhult J. Incidence and risk factors for injuries to the recurrent laryngeal nerve during neck surgery in the moderate-volume setting. Langenbecks Arch Surg. 2014; 399(4): 509-515, doi: 10.1007/s00423-013-1154-6, indexed in Pubmed: 24402457.

16. Pacella CM, Mauri G. Is there a role for minimally invasive thermal ablations in the treatment of autonomously functioning thyroid nodules? Int J Hyperthermia. 2018; 34(5): 636-638, doi: 10.1080/02656736.2018.14 62537, indexed in Pubmed: 29793352.

17. Alexander EK, Pearce EN, Brent GA, et al. 2017 Guidelines of the American Thyroid Association for the Diagnosis and Management of Thyroid Disease During Pregnancy and the Postpartum. Thyroid. 2017; 27(3): 315-389, doi: 10.1089/thy.2016.0457, indexed in Pubmed: 28056690.

18. Li H, Zheng J, Luo J, et al. Congenital anomalies in children exposed to antithyroid drugs in-utero: a meta-analysis of cohort studies. PLoS One. 2015; 10(5): e0126610, doi: 10.1371/journal.pone.0126610, indexed in Pubmed: 25974033.

19. Ceccarelli C, Canale D, Battisti $P$, et al. Testicular function after 131I therapy for hyperthyroidism. Clin Endocrinol (Oxf). 2006; 65(4): 446-452, doi: 10.1111/j.1365-2265.2006.02613.x, indexed in Pubmed: 16984236.

20. Kohlhase KD, Korkusuz Y, Gröner D, et al. Bipolar radiofrequency ablation of benign thyroid nodules using a multiple overlapping shot technique in a 3-month follow-up. Int J Hyperthermia. 2016; 32(5): 511-516, doi: 10.3109/02656736.2016.1149234, indexed in Pubmed: 27126512. 
21. Zhang M, Luo Y, Zhang Y, et al. Efficacy and Safety of Ultrasound-Guided Radiofrequency Ablation for Treating Low-Risk Papillary Thyroid Microcarcinoma: A Prospective Study. Thyroid. 2016; 26(11): 1581-1587, doi: 10.1089/thy.2015.0471, indexed in Pubmed: 27445090.

22. Valcavi R, Piana S, Bortolan GS, et al. Ultrasound-guided percutaneous laser ablation of papillary thyroid microcarcinoma: a feasibility study on three cases with pathological and immunohistochemical evaluation. Thyroid. 2013; 23(12): 1578-1582, doi: 10.1089/thy.2013.0279, indexed in Pubmed: 23978269.

23. Shengnan H, Lin Y, Lili P, et al. Microwave Ablation of An Autonomous Functioning Thyroid Nodule in A Pregnant Patient: A Case Report. Adv U1trasound DiagnTher. 2019; 3(3): 136, doi: 10.37015/audt.2019.190824.

24. Cervelli R, Mazzeo S, Boni G, et al. Comparison between radioiodine therapy and single-session radiofrequency ablation of autonomously functioning thyroid nodules: A retrospective study. Clin Endocrinol (Oxf). 2019; 90(4): 608-616, doi: 10.1111/cen.13938, indexed in Pubmed: 30657603.

25. Zingrillo M, Torlontano M, Ghiggi MR, et al. Radioiodine and percutaneous ethanol injection in the treatment of large toxic thyroid nodule: a long-term study. Thyroid. 2000; 10(11): 985-989, doi: 10.1089/thy.2000.10.985, indexed in Pubmed: 11128727.

26. Døssing H, Bennedbaek FN, Bonnema SJ, et al. Randomized prospective study comparing a single radioiodine dose and a single laser therapy session in autonomously functioning thyroid nodules. Eur J Endocrinol. 2007; 157(1): 95-100, doi: 10.1530/EJE-07-0094, indexed in Pubmed: 17609407.

27. Nygaard B, Hegedüs L, Nielsen KG, et al. Long-term effect of radioactive iodine on thyroid function and size in patients with solitary autonomously functioning toxic thyroid nodules. Clin Endocrinol (Oxf). 1999; 50(2): 197-202, doi: 10.1046/j.1365-2265.1999.00635.x, indexed in Pubmed: 10396362.

28. Sung JY, Baek JH, Jung SoL, et al. Radiofrequency ablation for autonomously functioning thyroid nodules: a multicenter study. Thyroid 2015; 25(1): 112-117, doi: 10.1089/thy.2014.0100, indexed in Pubmed: 25320840 .
29. Bernardi S, Stacul F, Michelli A, et al. 12-month efficacy of a single radiofrequency ablation on autonomously functioning thyroid nodules. Endocrine. 2017; 57(3): 402-408, doi: 10.1007/s12020-016-1174-4, indexed in Pubmed: 27848197.

30. Cerbone G, Spiezia S, Colao A, et al. Percutaneous ethanol injection under Power Doppler ultrasound assistance in the treatment of autonomously functioning thyroid nodules. J Endocrinol Invest. 1999; 22(10): 752-759, doi: 10.1007/BF03343640, indexed in Pubmed: 10614524

31. Yano Y, Sugino K, Akaishi J, et al. Treatment of autonomously functioning thyroid nodules at a single institution: radioiodine therapy, surgery, and ethanol injection therapy. Ann Nucl Med. 2011; 25(10): 749-754 doi: 10.1007/s12149-011-0526-7, indexed in Pubmed: 21971604.

32. Ross DS, Ridgway EC, Daniels GH. Successful treatment of solitary toxic thyroid nodules with relatively low-dose iodine-131, with low prevalence of hypothyroidism. Ann Intern Med. 1984; 101(4): 488-490, doi: 1 0.7326/0003-4819-101-4-488, indexed in Pubmed: 6476634.

33. Goldstein R, Hart IR. Follow-up of solitary autonomous thyroid nodules treated with 131I. N Engl J Med. 1983; 309(24): 1473-1476, doi: 10.1056/NEJM198312153092401, indexed in Pubmed: 6646172.

34. Mariotti S, Martino E, Francesconi M, et al. Serum thyroid autoantibodies as a risk factor for development of hypothyroidism after radioactive iodine therapy for single thyroid 'hot' nodule. Acta Endocrinol (Copenh). 1986; 113(4): 500-507, doi: 10.1530/acta.0.1130500, indexed in Pubmed: 3788420

35. Rho MHo, Kim DW, Hong HP, et al. Diagnostic value of antithyroid peroxidase antibody for incidental autoimmune thyroiditis based on histopathologic results. Endocrine. 2012; 42(3): 647-652, doi: 10.1007/s12020-012-9695-y, indexed in Pubmed: 22581205.

36. Takasu N, Yoshimura Noh J. Hashimoto's thyroiditis: TGAb, TPOAb $\mathrm{TRAb}$ and recovery from hypothyroidism. Expert Rev Clin Immunol. 2008; 4(2): 221-237, doi: 10.1586/1744666X.4.2.221, indexed in Pubmed: 20477052

37. Ramanathan P, Patel RB, Subrahmanyam N, et al. Visualization of suppressed thyroid tissue by technetium-99m-tertiary butyl isonitrile: an alternative to post-TSH stimulation scanning. J Nucl Med. 1990; 31(7): 1163-1165, indexed in Pubmed: 2362195 